Research Article

\title{
Negative Energy Solutions for a New Fractional $p(x)$-Kirchhoff Problem without the (AR) Condition
}

\author{
Weichun Bu $\mathbb{D}^{1},{ }^{1}$ Tianqing An $\mathbb{D}^{1},{ }^{1}$ Guoju Ye ${ }^{D},{ }^{1}$ and Said Taarabti ${ }^{2}$ \\ ${ }^{1}$ College of Science, Hohai University, Nanjing 210098, China \\ ${ }^{2}$ Laboratory of Systems Engineering and Information Technologies, National School of Applied Sciences of Agadir, \\ Ibn Zohr University, Morocco
}

Correspondence should be addressed to Weichun Bu; yangice0301@126.com

Received 9 September 2020; Accepted 11 March 2021; Published 23 March 2021

Academic Editor: Maria Alessandra Ragusa

Copyright (c) 2021 Weichun Bu et al. This is an open access article distributed under the Creative Commons Attribution License, which permits unrestricted use, distribution, and reproduction in any medium, provided the original work is properly cited.

In this paper, we investigate the following Kirchhoff type problem involving the fractional $p(x)$-Laplacian operator. $\left\{\begin{array}{l}\left(a-b \int_{\Omega \times \Omega}\left(|u(x)-u(y)|^{p(x, y)}|p(x, y)| x-\left.y\right|^{N+s p(x, y)}\right) d x d y\right) \mathscr{L} u=\lambda|u|^{q(x)-2} u+f(x, u) \mathrm{x} \in \Omega \text {, where } \Omega \text { is a bounded domain in } \\ u=0 \mathrm{x} \in \partial \Omega,\end{array}\right.$ $\mathbb{R}^{N}$ with Lipschitz boundary, $a \geq b>0$ are constants, $p(x, y)$ is a function defined on $\bar{\Omega} \times \bar{\Omega}, s \in(0,1)$, and $q(x)>1, \mathscr{L} u$ is the fractional $p(x)$-Laplacian operator, $N>s p(x, y)$, for any $(x, y) \in \bar{\Omega} \times \bar{\Omega}, p(x)^{*}=(p(x, x) N) /(N-s p(x, x)), \lambda$ is a given positive parameter, and $f$ is a continuous function. By using Ekeland's variational principle and dual fountain theorem, we obtain some new existence and multiplicity of negative energy solutions for the above problem without the Ambrosetti-Rabinowitz ((AR) for short) condition.

\section{Introduction and the Main Results}

In this article, we investigate the existence and multiplicity of solutions for the Kirchhoff type problem involving the fractional $p(x)$-Laplacian operator. More precisely, we consider the following problem $\left(P_{\lambda}\right)$ :

$$
\left(P_{\lambda}\right):\left\{\begin{array}{l}
\left(a-b \int_{\Omega \times \Omega} \frac{|u(x)-u(y)|^{p(x, y)}}{p(x, y)|x-y|^{N+s p(x, y)}} d x d y\right) \mathscr{L} u=\lambda|u|^{q(x)-2} u+f(x, u) \mathrm{x} \in \Omega, \\
u=0 \quad \mathrm{x} \in \partial \Omega
\end{array}\right.
$$

where $\Omega$ is a bounded domain in $\mathbb{R}^{N}$ with Lipschitz boundary and $a \geq b>0$ are constants, $s \in(0,1)$ and $N>s p(x, y)$ for all $(x, y) \in \bar{\Omega} \times \bar{\Omega}$. $\lambda$ is a given positive parameter, and $f$ is a continuous function. The operator $\mathscr{L} u=(-\Delta)_{p(\cdot)}^{s} u$ is the fractional $p(x)$-Laplacian operator, which is defined by

$$
\mathscr{L} u:=P . V . \int_{\Omega} \frac{|u(x)-u(y)|^{p(x, y)-2}(u(x)-u(y))}{|x-y|^{N+s p(x, y)}} d y, x \in \Omega,
$$

where P.V. stands for the Cauchy principal value. Especially, when $p(\cdot) \equiv$ constant, the operator $\mathscr{L} u=(-\Delta)_{p(\cdot)}^{s} u$ reduced to the usual fractional $p$-Laplace operator. In the past few decades, many people studied the fractional $p$-Laplace operator problem, and we refer the readers to [1-9]. We point out that Behboudi et al. studied the existence of mountain pass solution for the nonlocal fractional $(p, q)$-Laplacian problem in [10] by using the variational method. The more general problem is the following $p(x)$-Laplacian equation

$$
\left\{\begin{array}{l}
(-\Delta)_{p(x)}^{s} u=f(x, u) \text { in } \Omega, \\
u=0 \quad \text { in } \mathbb{R}^{N} \backslash \Omega
\end{array}\right.
$$


The study for this problem and the related knowledge on fractional Sobolev spaces with variable exponent please refer to $[11,12]$ and the references therein.

In the recent years, the fractional calculus and the related problems are studied widely, see for example, the literature $[13,14]$. The elementary knowledge of fractional derivative and integral can be found in $[15,16]$. Especially, the Caputo derivative is discussed in [17]. These references will be helpful for our research later.

The following Kirchhoff equation was introduced by Kirchhoff in [18]:

$$
\rho \frac{\partial^{2} u}{\partial t^{2}}-\left(\frac{p_{0}}{h}+\frac{E}{2 L} \int_{0}^{L}\left|\frac{\partial u}{\partial t}\right|^{2} d x\right) \frac{\partial^{2} u}{\partial x^{2}}=0,
$$

where $\rho, p_{0}, h, E, L$ are constants that have physical meaning. We call the problem $\left(P_{\lambda}\right)$ a new problem of Kirchhoff type because it contains the new Kirchhoff term:

$$
a-b \int_{\Omega \times \Omega} \frac{|u(x)-u(y)|^{p(x, y)}}{p(x, y)|x-y|^{N+s p(x, y)}} d x d y,
$$

which makes the problem $P_{\lambda}$ more interesting, meaningful, and difficult. Up to now, the study on the Kirchhoff type problems involving $p(x)$-Laplacian operator and the fractional $p(x)$-Laplacian operator is very active, see [19-23]. Many results concerning the existence and multiplicity of solutions have been appeared. Especially, [24] proved the existence of two weak solutions by using the variational methods in Orlicz-Sobolev spaces, [25] studied a class of Kirchhoff nonlocal fractional equations, and obtained the existence of three solutions. We also mention that [26] discussed a class of $p$-Kirchhoff equations via the fountain theorem and dual fountain theorem, and [27] studied the existence of nonnegative solutions for a Kirchhoff type problem driven by a nonlocal integrodifferential operator.

It is well known that the (AR) condition plays an important role in verifying the Palais-Smale condition. However, there are a lot of functions not satisfying this condition. Hence, many people pay attention to find the new reasonable conditions instead of the (AR) condition, see [28-31] and the references therein. Motivated by these work, we use Ekeland's variational principle and dual fountain theorem to study the existence and multiplicity of negative energy solutions for a new fractional $p(x)$-Kirchhoff problem without the (AR) condition. Our results generalize the related work in two ways. Firstly, we deal with the problem $\left(P_{\lambda}\right)$ in the fractional framework and [32] consider only the integer framework. Secondly, compare to [33], we add a new Kirchhoff function and consider the case of variable exponents.

Throughout this paper, the nonlinearity $f(x, t): \Omega \times \mathbb{R}$

$\longrightarrow \mathbb{R}$ is a Carathe' odory function satisfying

(f1): There exists a positive constant $C$ such that

$$
|f(x, t)| \leq C\left(1+|t|^{r(x)-1}\right) \text {, for all }(x, t) \in \Omega \times \mathbb{R} \text {, }
$$

where $1<r(x)<p(x)^{*}=(p(x, x) N) /(N-s p(x, x))$; (f2): $\lim _{|t| \longrightarrow 0}\left(f(x, t) t /|t|^{p^{+}}\right)=0$, for $x \in \Omega$ uniformly;

(f3): $\lim _{|t| \longrightarrow \infty} F(x, t) /|t|^{2 p^{+}}=+\infty, x \in \Omega$, where $F(x, t)$ $=\int_{0}^{t} f(x, s) d s \geq 0$

(f4):): There exists positive constants $\theta_{0}$ such that $F(x, t)$ $\leq\left(1 / p^{+}\right) f(x, t) t+\theta_{0}|t|^{2 p^{-}}$, for all $(x, t) \in \Omega \times \mathbb{R}$;

(f5): $f(x,-t)=-f(x, t)$, for all $(x, t) \in \Omega \times \mathbb{R}$.

Definition 1 . We say $u \in X_{0}$ is a weak solution of problem $\left(P_{\lambda}\right)$, if

$$
\begin{aligned}
& \left(a-b \int_{\Omega \times \Omega} \frac{|u(x)-u(y)|^{p(x, y)}}{p(x, y)|x-y|^{N+s p(x, y)}} d x d y\right) \\
& \quad \times \int_{\Omega \times \Omega} \frac{|u(x)-u(y)|^{p(x, y)-2}(u(x)-u(y))(\varphi(x)-\varphi(y))}{|x-y|^{N+s p(x, y)}} d x d \\
& =\lambda \int_{\Omega}|u|^{q(x)-2} u \varphi d x+\int_{\Omega} f(x, u) \varphi d x
\end{aligned}
$$

for any $\varphi \in X_{0}$, where $X_{0}$ will be introduced in Section 2 .

It is well known that a weak solution for problem $\left(P_{\lambda}\right)$ is a critical point of the following energy functional $I$ defined on $X_{0}$ by

$$
\begin{aligned}
I(u):= & a \int_{\Omega \times \Omega} \frac{|u(x)-u(y)|^{p(x, y)}}{p(x, y)|x-y|^{N+s p(x, y)}} d x d y \\
& -\frac{b}{2}\left(\int_{\Omega \times \Omega} \frac{|u(x)-u(y)|^{p(x, y)}}{p(x, y)|x-y|^{N+s p(x, y)}} d x d y\right)^{2} \\
& -\lambda \int_{\Omega} \frac{1}{q(x)}|u|^{q(x)} d x-\int_{\Omega} F(x, u) d x
\end{aligned}
$$

for all $u \in X_{0}$. Moreover, we have

$$
\begin{aligned}
\left\langle I^{\prime}(u), \varphi\right\rangle:= & \left(a-b \int_{\Omega \times \Omega} \frac{|u(x)-u(y)|^{p(x, y)}}{p(x, y)|x-y|^{N+s p(x, y)}} d x d y\right) \\
& \times \int_{\Omega} \frac{|u(x)-u(y)|^{p(x, y)-2}(u(x)-u(y))(\varphi(x)-\varphi(y))}{|x-y|^{N+s p(x, y)}} d x \\
& -\lambda \int_{\Omega}|u|^{q(x)-2} u \varphi d x-\int_{\Omega} f(x, u) \varphi d x,
\end{aligned}
$$

for any $u, \varphi \in X_{0}$. Under our assumptions, $I$ is well defined in $X_{0}$ and $I \in C^{1}\left(X_{0}, \mathbb{R}\right)$. We say that a weak solution $u$ for problem $\left(P_{\lambda}\right)$ is a negative energy solution if the energy $I(u)<0$.

In order to reduce our statements, we define the function space

$C_{+}(\bar{\Omega}):=\{H(x): H(x) \in C(\bar{\Omega}, \mathbb{R}), 1<H(x)<$ ofor all $x \in \bar{\Omega}\}$,

and $q^{-}, q^{+}, p^{-}, p^{+}, r^{-}$will be introduced in Section 2 . The main results of this paper are as follows. 
Theorem 2. Assume that the function $f$ satisfies (f1)-(f4) and

$$
1<q^{-}<q^{+}<p^{-}<p^{+}<\sqrt{2} p^{-}<2 p^{-}<r^{-}<2 p^{+}<p^{*}(x) .
$$

Then, there exists a $\lambda^{*}>0$ such that for any $0<\lambda<\lambda^{*}$, the problem $\left(P_{\lambda}\right)$ has at least one solution $u_{0}$ with negative energy.

Theorem 3. Assume that the function $f$ satisfies (f1)-(f5) and

$$
1<q^{-}<q^{+}<p^{-}<p^{+}<\sqrt{2} p^{-}<2 p^{-}<r^{-}<2 p^{+}<p^{*}(x) .
$$

Then, for any $\lambda>0$, problem $\left(P_{\lambda}\right)$ has infinitely many solutions $\left\{u_{n}\right\}$ in $X_{0}$ with negative energy converging to 0 .

The rest of this paper is organized as follows. In Section 2, some basic properties of the variable exponent fractional Sobolev spaces and Lebesgue spaces are given. In Section 3, it is proved that the functional satisfies the Cerami compactness condition in certain energy levels. In Section 4, by using Ekeland's variational principle, we give the proof of Theorem 2. Finally, in Section 5, we prove Theorem 3 by using the dual fountain theorem.

Throughout this paper, for simplicity, we use letters $C_{i}$ $(i=1,2, \cdots, N)$ to denote positive constants in different cases, and we will specify them whenever it is necessary.

\section{Preliminary Results}

In this section, we recall some preliminary results of generalized Lebesgue spaces $L^{q(x)}(\Omega)$ with variable exponent and generalized fractional Sobolev spaces $W^{s, h(x), p(x, y)}(\Omega)$ which will be used later. The readers can consult [34-36] and the references therein for more details. Let $s \in(0,1)$ and $\Omega$ be a bounded domain in $\mathbb{R}^{N}$ with Lipschitz boundary, and $q(x)$, $h(x), p(x, y)$ be continuous functions satisfying

$$
\begin{gathered}
1<q^{-}:=\min _{x \in \bar{\Omega}} q(x) \leq q(x) \leq q^{+}:=\max _{x \in \bar{\Omega}} q(x)<\infty, \\
1<h^{-}:=\min _{x \in \bar{\Omega}} h(x) \leq h(x) \leq h^{+}:=\max _{x \in \bar{\Omega}} h(x)<\infty .
\end{gathered}
$$

We assume that $p(x, y)$ is symmetric; that is, $p(x, y)=$ $p(y, x)$ for all $(x, y) \in \bar{\Omega} \times \bar{\Omega}$, such that

$1<p^{-}:=\min _{(x, y) \in \bar{\Omega} \times \bar{\Omega}} p(x, y) \leq p(x, y) \leq p^{+}:=\max _{(x, y) \in \bar{\Omega} \times \bar{\Omega}} p(x, y)<\infty$.

For any $q(x) \in C_{+}(\bar{\Omega})$, we introduce the variable exponent Lebesgue space as

$$
L^{q(x)}(\Omega):=\left\{u: u \text { is measurable, } \int_{\Omega}|u|^{q(x)} d x<\infty\right\}
$$

endowed with the so-called Luxemburg norm

$$
|u|_{q(x)}:=\inf \left\{\mu>0: \int_{\Omega}\left|\frac{u}{\mu}\right|^{q(x)} d x \leq 1\right\} .
$$

Define a mapping $\rho: L^{q(x)}(\Omega) \longrightarrow \mathbb{R}$ by

$$
\rho_{q(x)}(u):=\int_{\Omega}|u|^{q(x)} d x .
$$

Lemma 4 (See [37]). The space $\left(L^{q(x)}(\Omega),|u|_{q(x)}\right)$ is separable, uniformly convex, and reflexive and its conjugate space is $\left(L^{q^{\prime}(x)}(\Omega),|u|_{q}^{\prime(x)}\right)$, where $q^{\prime}(x)$ is the conjugate function of $q(x)$, i.e.,

$$
\frac{1}{q(x)}+\frac{1}{q^{\prime}(x)}=1, \text { for all } x \in \Omega \text {, }
$$

for all $u \in L^{q(x)}(\Omega), v \in L^{q^{\prime}(x)}(\Omega)$, and the Hölder type inequality

$$
\left|\int_{\Omega} u v d x\right| \leq\left(\frac{1}{q^{-}}+\frac{1}{\left(q^{\prime}\right)^{-}}\right)|u|_{q(x)}|v|_{q}^{\prime(x)} \leq 2|u|_{q(x)}|v|_{q}^{\prime(x)}
$$

holds.

Note that for any function $q_{1}(x), q_{2}(x) \in C_{+}(\bar{\Omega})$ and $q_{1}(x)<q_{2}(x)$, there exists a continuous embedding $L^{q_{2}(x)}$ $(\Omega)^{\hookrightarrow} L^{q_{1}(x)}(\Omega)$ for any $x \in \bar{\Omega}$.

Lemma 5 (see [38]). Suppose that $u_{n}, u \in L^{q(x)}(\Omega)$. Then, the following properties hold

$$
\begin{gathered}
|u|_{q(x)}>1 \Rightarrow|u|_{q(x)}^{q^{-}} \leq \rho_{q(x)}(u) \leq|u|_{q(x)}^{q^{+}}, \\
|u|_{q(x)}<1 \Rightarrow|u|_{q(x)}^{q^{+}} \leq \rho_{q(x)}(u) \leq|u|_{q(x)}^{q^{-}}, \\
\left.|u|_{q(x)}<1(\text { respectively, }=1,>1) \Leftrightarrow \rho_{q(x)}(u)<1(\text { respectively },=1,>1)\right), \\
\left|u_{n}\right|_{q(x)} \longrightarrow 0(\text { respectively, } \longrightarrow \infty) \Leftrightarrow \rho_{q(x)}\left(u_{n}\right) \longrightarrow 0(\text { respectively }, \longrightarrow \infty), \\
\lim _{n \longrightarrow \infty}\left|u_{n}-u\right|_{q(x)}=0 \Leftrightarrow \lim _{n \longrightarrow \infty} \rho_{q(x)}\left(u_{n}-u\right)=0 .
\end{gathered}
$$

The fractional Sobolev spaces with variable exponent $X=W^{s, h(x), p(x, y)}(\Omega)$ are defined by

$$
W^{s, h(x), p(x, y)}(\Omega):=\left\{u: u \in L^{h(x)}(\Omega), \int_{\Omega \times \Omega} \frac{|u(x)-u(y)|^{p(x, y)}}{\mu^{p(x, y)}|x-y|^{N+s p(x, y)}} d x d y<\infty, \lambda>0\right\} .
$$


For $u \in X$, let

$$
[u]_{s, p(x, y)}:=\inf \left\{\mu>0, \int_{\Omega \times \Omega} \frac{|u(x)-u(y)|^{p(x, y)}}{\mu^{p(x, y)}|x-y|^{N+s p(x, y)}} d x d y \leq 1\right\} .
$$

Define the corresponding variable exponent norm

$$
\|u\|_{X}:=|u|_{h(x)}+[u]_{s, p(x, y)}
$$

and set

$$
\rho_{p(x . y)}(u):=\int_{\Omega \times \Omega} \frac{|u(x)-u(y)|^{p(x, y)}}{|x-y|^{N+s p(x, y)}} d x d y .
$$

Then, the $\left(X,\|\cdot\|_{X}\right)$ becomes an uniformly convex and reflexive Banach spaces (see [39]). Let $X_{0}=W_{0}^{s, h(x), p(x, y)}(\Omega)$ denote the closure of $C_{0}^{\infty}(\Omega)$ in $X$ endowed with the norm

$$
\|u\|_{X_{0}}:=[u]_{s, p(x, y)}, \text { for all } u \in X_{0} \text {, }
$$

and then $\left(X_{0},\|\cdot\|_{X_{0}}\right)$ is also an uniformly convex and reflexive Banach spaces. $X_{0}^{*}$ is the dual spaces of $X_{0}$.

Theorem 6 (see [39]). Let $\Omega \in \mathbb{R}^{N}$ be a smooth bounded domain and $s \in(0,1), h(x), p(x, y)$ be continuous variable exponents with $N>\operatorname{sp}(x, y)$ for $(x, y) \in \Omega \times \Omega$ and $h(x)>$ $p(x, x)$ for $x \in \bar{\Omega}$, and (13) and (15) be satisfied. Assume that $r^{\prime}: \bar{\Omega} \longrightarrow(1, \infty)$ is a continuous function and $r^{\prime^{-}}=$ $\min _{x \in \bar{\Omega}^{\prime}} r^{\prime}(x)$ such that

$$
p(x)^{*}=\frac{p(x, x) N}{N-s p(x, x)}>r^{\prime}(x) \geq r^{\prime^{-}}>1,
$$

for $x \in \bar{\Omega}$. Then, there exists a constant $C_{0}=C_{0}\left(N, s, p, h, r^{\prime}\right.$, $\Omega$ ) such that for any $u \in X$,

$$
|u|_{r}^{\prime(x)} \leq C_{0}\|u\|_{X}
$$

Thus, the spaces $X$ is continuously embedded in $L^{r^{\prime}(x)}(\Omega)$ for any $r^{\prime}(x) \in\left(1, p^{*}\right)$. Furthermore, this embedding is compact. If $u \in X_{0}$, then there exists a constant $C_{0}=C_{0}(N, s, p, h$ $\left., r^{\prime}, \Omega\right)$ such that

$$
|u|_{r}^{\prime(x)} \leq C_{0}[u]_{s, p(x, y)}
$$

Lemma 7 (See [40]). For all $u, \varphi \in X_{0}$, define the functional $\mathscr{L} u: X_{0} \longrightarrow X_{0}^{*}$ by

$$
\langle\mathscr{L} u, \varphi\rangle:=\int_{\Omega \times \Omega} \frac{|u(x)-u(y)|^{p(x, y)-2}(u(x)-u(y))(\varphi(x)-\varphi(y))}{|x-y|^{N+s p(x, y)}} d x d y .
$$

Then,
(1) $\mathscr{L} u$ is a bounded and strictly monotone operator

(2) $\mathscr{L} u$ is a mapping of type $\left(S_{+}\right)$, i.e., if $u_{n} \longrightarrow u$ in $X_{0}$ and $\limsup _{n \rightarrow \infty}\left\langle\mathscr{L} u_{n}-\mathscr{L} u, u_{n}-u\right\rangle \leq 0$, then $u_{n}$ $\longrightarrow u$ in $X_{0}$

(3) $\mathscr{L} u: X_{0} \longrightarrow X_{0}^{*}$ is a homeomorphism

Lemma 8 (see [41]).

(1) If $1 \leq\|u\|_{X_{0}}<\infty$, then

$$
\|u\|_{X_{0}}^{p^{-}} \leq \rho_{p(x . y)}(u) \leq\|u\|_{X_{0}}^{p^{+}} .
$$

(2) If $\|u\|_{X_{0}}<1$, then

$$
\|u\|_{X_{0}}^{p^{+}} \leq \rho_{p(x . y)}(u) \leq\|u\|_{X_{0}}^{p^{-}} .
$$

We state Ekeland's variational principle and dual fountain theorem which will be used in the proofs of Theorems 2 and 3.

Theorem 9 (Ekeland's variational principle, see [42]). Let $X_{0}$ be a complete metric spaces with metric $d$, and let $I: X_{0}$ $\mapsto(-\infty,+\infty]$ be a lower semicontinuous function, bounded from below and not identical to $+\infty$. Let $\varepsilon_{0}$ be given and $U$ $\in X_{0}$ be such that

$$
I(U) \leq \inf _{X_{0}} I+\varepsilon_{0}
$$

Then, there exists $V \in X_{0}$ such that

$$
I(U) \leq I(V), d(U, V) \leq 1,
$$

and for each $W \in X_{0}$, one has

$$
I(U) \leq I(W)+\varepsilon_{0} d(V, W) .
$$

The space $X_{0}$ is a separable and reflexive real Banach space, and there exists $\left\{e_{j}\right\} \in X_{0}$ and $\left\{e_{j}^{*}\right\} \in X_{0}^{*}$ such that $X_{0}$ $=\operatorname{span}\left\{e_{j}: \bar{j}=1,2, \cdots\right\}, X_{0}^{*}=\operatorname{span}\left\{e_{j}^{*}: \bar{j}=1,2, \cdots\right\}$ and

$$
\left\langle e_{j}^{*}, e_{i}\right\rangle=\left\{\begin{array}{l}
1, i \neq j ; \\
0, i=j .
\end{array}\right.
$$

Set $X_{i}=\operatorname{span}\left\{e_{i}\right\}$, we define $A_{k}=\oplus_{i=1}^{k} X_{i}, B_{k}=\oplus_{i=k}^{\bar{\alpha}} X_{i}$.

Theorem 10 (Dual Fountain Theorem, see [43]). Assume that $I \in C^{1}\left(X_{0}, \mathbb{R}\right)$ satisfies $I(-u)=I(u)$, and for every $k \geq k_{0}$, there exist $\rho_{k}>\gamma_{k}>0$ such that

(B1) $a_{k}=\inf \left\{I(u): u \in B_{k} ;\|u\|=\rho_{k}\right\} \geq 0$;

(B2) $b_{k}=\max \left\{I(u): u \in A_{k} ;\|u\|=\gamma_{k}\right\}<0$;

(B3) $d_{k}=\inf \left\{I(u): u \in B_{k} ;\|u\| \leq \gamma_{k}\right\} \longrightarrow 0$, ask $\longrightarrow \infty$;

(B4) I satisfies the $(\mathrm{Ce})_{c}^{*}$ condition for every $c \in\left[d_{k_{0}}, 0\right]$. 
Then, I has a sequence of negative critical values converging to 0 .

\section{Cerami Compactness Condition}

We discuss the compactness properties of our energy functional $I$ related to the $(\mathrm{Ce})_{c}$ condition and $(\mathrm{Ce})_{c}^{*}$ condition.

Definition 11. Let $I \in C^{1}(X, \mathbb{R})$, and we say that $I$ satisfies the Cerami condition at the level $c \in \mathbb{R}\left((\mathrm{Ce})_{c}\right.$ for short), if any sequence $\left\{u_{n}\right\} \subset X_{0}$ with

$$
I\left(u_{n}\right) \longrightarrow c,\left(1+\left\|u_{n}\right\|_{X_{0}}\right) I^{\prime}\left(u_{n}\right) \longrightarrow 0, \text { asn } \longrightarrow \infty
$$

possesses a convergent subsequence in $X_{0}$.

Definition 12. Let $I \in C^{1}(X, \mathbb{R})$, and we say that $I$ satisfies $(C e)_{c}^{*}$ condition at the level $c \in \mathbb{R}\left((C e)_{c}^{*}\right.$ for short), if any sequence $\left\{u_{n_{j}}^{-}\right\}_{n_{j}} \subset X_{0}$, namely, $u_{n_{j}}^{-} \in A_{n_{j}}$, with

$$
I\left(u_{n_{j}}^{-}\right) \longrightarrow c,\left(1+\left\|u_{n_{j}}^{-}\right\|_{X_{0}}\right) I^{\prime}\left(u_{n_{j}}^{-}\right) \longrightarrow 0, a s n_{j} \longrightarrow \infty
$$

possesses a convergent subsequence in $X_{0}$.

Assume that $\left\{u_{n}\right\}$ is a bounded sequence in $X_{0}$. By Theorem 6, there exists $u \in X_{0}$ such that

$$
u_{n} \rightarrow u \text { in } X_{0}, u_{n} \longrightarrow \text { ua.e.in } \Omega, u_{n} \longrightarrow u \text { in } L^{q(x)}(\Omega) \text {. }
$$

Lemma 13. Let $u_{n}, u, v \in X_{0}$ such that (38) holds. Then, passing to a subsequence, the following properties hold

$$
\begin{gathered}
\lim _{n \longrightarrow \infty} \int_{\Omega}\left|u_{n}\right|^{q(x)-2} u_{n}\left(u_{n}-u\right) d x=0, \\
\lim _{n \longrightarrow \infty} \int_{\Omega} f\left(x, u_{n}\right)\left(u_{n}-u\right) d x=0 .
\end{gathered}
$$

Proof.

(i) By Hölder's inequality (Lemma 4),Theorem 6, and (38), we have

$$
\begin{aligned}
& \left.\left.\left|\int_{\Omega}\right| u_{n}\right|^{q(x)-2} u_{n}\left(u_{n}-u\right) d x\left|\leq \int_{\Omega}\right| u_{n}\right|^{q(x)-1}\left|u_{n}-u\right| d x \\
& \leq\left.\left.|| u_{n}\right|^{q(x)-1}\right|_{q(x) / q(x)-1}\left|u_{n}-u\right|_{q(x)} \longrightarrow 0 \text {, as } n \longrightarrow \infty,
\end{aligned}
$$

and thus

$$
\lim _{n \longrightarrow \infty} \int_{\Omega}\left|u_{n}\right|^{q(x)-2} u_{n}\left(u_{n}-u\right) d x=0 .
$$

(ii) By virtue of conditions (f1) and (f2), for any $\varepsilon \in(0,1)$, there exists $C_{\varepsilon}>0$ such that

$$
\left|f\left(x, u_{n}\right)\right| \leq \varepsilon\left|u_{n}\right|^{p^{+}-1}+C_{\varepsilon}\left|u_{n}\right|^{r(x)-1},
$$

combining with Hölder's inequality, Theorem 6 and (38),and it follows that

$$
\begin{aligned}
& \left|\int_{\Omega} f\left(x, u_{n}\right)\left(u_{n}-u\right) d x\right| \\
& \leq \int_{\Omega}\left(\varepsilon\left|u_{n}\right|^{p^{+}-1}\left|u_{n}-u\right|+C_{\varepsilon}\left|u_{n}\right|^{r(x)-1}\left|u_{n}-u\right|\right) d x \\
& \leq\left.\left.\varepsilon|| u_{n}\right|^{p^{-}-1}\right|_{p^{+} / p^{+}-1}\left|u_{n}-u\right|_{p^{+}} \\
& \quad+\left.\left.C_{\varepsilon}|| u_{n}\right|^{r(x)-1}\right|_{r(x) / r(x)-1}\left|u_{n}-u\right|_{r(x)} \longrightarrow 0, \text { as } n \longrightarrow \infty,
\end{aligned}
$$

which implies that

$$
\lim _{n \longrightarrow \infty} \int_{\Omega} f\left(x, u_{n}\right)\left(u_{n}-u\right) d x=0
$$

The following lemma about the $(\mathrm{Ce})_{c}$ condition will play an important role in the proof of our main results.

Lemma 14. Let the function $f$ satisfy $(f 1),(f 3)$, and (f4), then the energy functional I satisfies the $(\mathrm{Ce})_{c}$ condition, where precisely $c<a^{2} / 2 b$.

Proof. Step 1. We prove that $\left\{u_{n}\right\}$ is bounded in $X_{0}$. Let $\left\{u_{n}\right\}$ be a $(C e)_{c}$ sequence, $I\left(u_{n}\right) \longrightarrow c,\left(1+\left\|u_{n}\right\|_{X_{0}}\right) I^{\prime}\left(u_{n}\right) \longrightarrow 0$, which implies that

$$
c=I\left(u_{n}\right)+o(1),\left\langle I^{\prime}\left(u_{n}\right), u_{n}\right\rangle=o(1)
$$

where $o(1) \longrightarrow 0$ as $n \longrightarrow \infty$.

We claim that $\left\{u_{n}\right\}$ is a bounded sequence. Suppose to the contrary that

$$
\left\|u_{n}\right\|_{X_{0}} \longrightarrow \infty \text {,as } n \longrightarrow \infty
$$

Denote $\omega_{n}:=u_{n} /\left\|u_{n}\right\|_{X_{0}}$, then $\omega_{n} \in X_{0}$ with $\left\|\omega_{n}\right\|_{X_{0}}=1$. Up to subsequences, for some $\omega \in X_{0}$, we get $\omega_{n} \rightarrow \omega$ in $X_{0}$, $\omega_{n} \longrightarrow \omega$ a.e. in $\Omega, \omega_{n} \longrightarrow \omega$ in $L^{q(x)}(\Omega)$. There are only two cases need to be discussed $\omega=0$ and $\omega \neq 0$. 
First, let us consider the case $\omega=0$. In view of (f4), (8), (9), (45), (46), and Theorem 6, we obtain

$$
\begin{aligned}
& 0=\frac{1}{\left\|u_{n}\right\|_{X_{0}}^{2 p^{-}}}\left[I\left(u_{n}\right)-\frac{1}{p^{+}}\left\langle I^{\prime}\left(u_{n}\right), u_{n}\right\rangle\right] \\
& =\frac{1}{\left\|u_{n}\right\|_{X_{0}}^{2 p^{-}}}\left[a \int_{\Omega \times \Omega} \frac{\left|u_{n}(x)-u_{n}(y)\right|^{p(x, y)}}{p(x, y)|x-y|^{N+s p(x, y)}} d x d y\right. \\
& -\frac{b}{2}\left(\int_{\Omega \times \Omega} \frac{\left|u_{n}(x)-u_{n}(y)\right|^{p(x, y)}}{p(x, y)|x-y|^{N+s p(x, y)}} d x d y\right)^{2} \\
& -\lambda \int_{\Omega} \frac{1}{q(x)}\left|u_{n}\right|^{q(x)} d x-\int_{\Omega} F\left(x, u_{n}\right) d x \\
& -\frac{a}{p^{+}} \int_{\Omega \times \Omega} \frac{\left|u_{n}(x)-u_{n}(y)\right|^{p(x, y)}}{|x-y|^{N+s p(x, y)}} d x d y \\
& +\frac{b}{p^{+}} \int_{\Omega \times \Omega} \frac{\left|u_{n}(x)-u_{n}(y)\right|^{p(x, y)}}{p(x, y)|x-y|^{N+s p(x, y)}} d x d y \\
& \times \int_{\Omega \times \Omega} \frac{\left|u_{n}(x)-u_{n}(y)\right|^{p(x, y)}}{|x-y|^{N+s p(x, y)}} \mathrm{d} x d y \\
& \left.+\frac{\lambda}{p^{+}} \int_{\Omega}\left|u_{n}\right|^{q(x)} d x+\frac{1}{p^{+}} \int_{\Omega} f\left(x, u_{n}\right) u_{n} d x\right] \\
& \geq \frac{1}{\left\|u_{n}\right\|_{X_{0}}^{2 p^{-}}}\left[\frac{a}{p^{+}} \int_{\Omega \times \Omega} \frac{\left|u_{n}(x)-u_{n}(y)\right|^{p(x, y)}}{|x-y|^{N+s p(x, y)}} d x d y\right. \\
& -\frac{b}{2 p^{-2}}\left(\int_{\Omega \times \Omega} \frac{\left|u_{n}(x)-u_{n}(y)\right|^{p(x, y)}}{|x-y|^{N+s p(x, y)}} d x d y\right)^{2} \\
& -\frac{\lambda}{\mathrm{q}^{-}} \int_{\Omega}\left|u_{n}\right|^{q(x)} d x-\int_{\Omega} F\left(x, u_{n}\right) d x \\
& -\frac{a}{p^{+}} \int_{\Omega \times \Omega} \frac{\left|u_{n}(x)-u_{n}(y)\right|^{p(x, y)}}{|x-y|^{N+s p(x, y)}} d x d y \\
& +\frac{b}{p^{+2}} \int_{\Omega \times \Omega} \frac{\left|u_{n}(x)-u_{n}(y)\right|^{p(x, y)}}{|x-y|^{N+s p(x, y)}} d x d y \\
& \times \int_{\Omega \times \Omega} \frac{\left|u_{n}(x)-u_{n}(y)\right|^{p(x, y)}}{|x-y|^{N+s p(x, y)}} d x d y \\
& \left.+\frac{\lambda}{p^{+}} \int_{\Omega}\left|u_{n}\right|^{q(x)} d x+\frac{1}{p^{+}} \int_{\Omega} f\left(x, u_{n}\right) u_{n} d x\right] \\
& \geq \frac{1}{\left\|u_{n}\right\|_{X_{0}}^{2 p^{-}}}\left[\left(\frac{b}{p^{+2}}-\frac{b}{2 p^{-2}}\right)\left\|u_{n}\right\|_{X_{0}}^{2 p^{-}}-\left(\frac{\lambda}{q^{-}}-\frac{\lambda}{p^{+}}\right) C_{q^{-}}\left\|u_{n}\right\|_{X_{0}}^{q^{-}}\right. \\
& \left.+\int_{\Omega}\left(\frac{1}{p^{+}} f\left(x, u_{n}\right) u_{n}-F\left(x, u_{n}\right)\right) d x\right] \\
& \geq \frac{1}{\left\|u_{n}\right\|_{X_{0}}^{2 p^{-}}}\left[\left(\frac{b}{p^{+2}}-\frac{b}{2 p^{-2}}\right)\left\|u_{n}\right\|_{X_{0}}^{2 p^{-}}-\left(\frac{\lambda}{q^{-}}-\frac{\lambda}{p^{+}}\right) C_{q^{-}}\left\|u_{n}\right\|_{X_{0}}^{q^{-}}\right. \\
& \left.-\theta_{0}\left\|u_{n}\right\|_{X_{0}}^{2 p^{-}} \int_{\Omega}\left|\omega_{n}\right|^{2 p^{-}} d x\right] \geq\left(\frac{b}{p^{+2}}-\frac{b}{2 p^{-2}}\right) \\
& -\left(\frac{\lambda}{q^{-}}-\frac{\lambda}{p^{+}}\right) C_{q^{-}}\left\|u_{n}\right\|_{X_{0}}^{q^{-}-2 p^{-}}-\theta_{0} \int_{\Omega}\left|\omega_{n}\right|^{2 p^{-}} d x,
\end{aligned}
$$

which implies $0>b / p^{+2}-b / 2 p^{-2}$, and this is a contradiction according to the conditions $p^{+}<\sqrt{2} p^{-}$and $q^{-}<2 p^{-}$(see (11)).

In the case of $\omega \neq 0$, setting $\Omega_{1}:=\{x \in \Omega: \omega \neq 0\}$, then $\lim _{n \longrightarrow \infty} \omega_{n}=\lim _{n \longrightarrow \infty} u_{n} /\left\|u_{n}\right\|_{X_{0}}=\omega \neq 0$, from (46) implies that $\left|u_{n}\right|=\left|\omega_{n}\right||| u_{n} \|_{X_{0}} \longrightarrow+\infty$, for $x \in \Omega_{1}$, we have $u_{n} \longrightarrow$ $\infty$ as $n \longrightarrow \infty$. In view of (f3), we see that

$$
\begin{aligned}
\frac{F\left(x, u_{n}\right)}{\left\|u_{n}\right\|_{X_{0}}^{2 p^{+}}} & =\frac{F\left(x, u_{n}\right)}{\left|u_{n}\right|^{2 p^{+}}} \frac{\left|u_{n}\right|^{2 p^{+}}}{\left\|u_{n}\right\|_{X_{0}}^{2 p^{+}}} \\
& =\frac{F\left(x, u_{n}\right)}{\left|u_{n}\right|^{2 p^{+}}}\left|\omega_{n}\right|^{2 p^{+}} \longrightarrow+\infty \text {, a.e.in } \Omega_{1}
\end{aligned}
$$

so, using Fatou's Lemma, we have

$$
\lim _{n \rightarrow \infty} \int_{\Omega_{1}} \frac{F\left(x, u_{n}\right)}{\left\|u_{n}\right\|_{X_{0}}^{2 p^{+}}} d x \longrightarrow+\infty \text {,a.e.in } \Omega_{1}
$$

From (f1) and (f3), there exist $M^{\prime}, M^{\prime \prime}, M>0$, such that $\left|F\left(x, u_{n}\right)\right| \leq M^{\prime}\left|u_{n}\right|^{r(x)}$ and $F\left(x, u_{n}\right) \geq-M^{\prime \prime}\left|u_{n}\right|^{2 p^{+}}$as $\left|u_{n}\right|$ $\longrightarrow \infty$, and then we have $F\left(x, u_{n}\right) \geq-M\left|u_{n}\right|^{r-}$, hence

$$
\int_{\Omega \backslash \Omega_{1}} \frac{F\left(x, u_{n}\right)}{\left\|u_{n}\right\|_{X_{0}}^{2 p^{+}}} d x \geq-\frac{\int_{\Omega \backslash \Omega_{1}} M\left|u_{n}\right|^{r-} d x}{\left\|u_{n}\right\|_{X_{0}}^{2 p^{+}}} \geq-\frac{M\left|u_{n}\right|^{r-}}{\left\|u_{n}\right\|_{X_{0}}^{2 p^{+}}},
$$

by (46), which implies that

$$
\liminf _{n \rightarrow \infty} \int_{\Omega \backslash \Omega_{1}} \frac{F\left(x, u_{n}\right)}{\left\|u_{n}\right\|_{X_{0}}^{2 p^{+}}} d x \geq 0
$$

Using (8), (45), (46), (49), (51), and Theorem 6, we obtain

$$
\begin{aligned}
0= & \lim _{n \longrightarrow \infty} \frac{-c+o(1)}{\left\|u_{n}\right\|_{X_{0}}^{2 p^{+}}}=\lim _{n \longrightarrow \infty} \frac{-I\left(u_{n}\right)}{\left\|u_{n}\right\|_{X_{0}}^{2 p^{+}}} \geq \lim _{n \longrightarrow \infty} \frac{1}{\left\|u_{n}\right\|_{X_{0}}^{2 p^{+}}} \\
& \cdot\left(-\frac{a}{p^{-}}\left\|u_{n}\right\|_{X_{0}}^{p^{+}}+\frac{b}{2\left(p^{+}\right)^{2}}\left\|u_{n}\right\|_{X_{0}}^{2 p^{-}}+\frac{\lambda C_{q}}{q^{+}}\left\|u_{n}\right\|_{X_{0}}^{q^{-}}+\int_{\Omega} F\left(x, u_{n}\right) d x\right) \\
\geq & -\frac{a}{p^{-}} \lim _{n \longrightarrow \infty}\left\|u_{n}\right\|_{X_{0}}^{p^{+}-2 p^{+}}+\frac{b}{2\left(p^{+}\right)^{2}} \lim _{n \longrightarrow \infty}\left\|u_{n}\right\|_{X_{0}}^{2 p^{-}-2 p^{+}} \\
& +\frac{\lambda C_{q}}{q^{+}} \lim _{n \longrightarrow \infty}\left\|u_{n}\right\|_{X_{0}}^{q^{-}-2 p^{+}}+\lim _{n \longrightarrow \infty} \int_{\Omega_{1}} \frac{F\left(x, u_{n}\right)}{\left\|u_{n}\right\|_{X_{0}}^{2 p^{+}}} d x \\
& +\liminf _{n \longrightarrow \infty} \int_{\Omega \backslash \Omega_{1}} \frac{F\left(x, u_{n}\right)}{\left\|u_{n}\right\|_{X_{0}}^{2 p^{+}}} d x \longrightarrow+\infty, \text { as } n \longrightarrow \infty,
\end{aligned}
$$

and this is a contradiction according to the condition $q^{-}<$ $p^{-}<p^{+}($see $(11))$. Then, the sequence $\left\{u_{n}\right\}$ is bounded in $X_{0}$.

Step 2. We prove that $\left\{u_{n}\right\}$ has a convergent subsequence in $X_{0}$. Since $\left(1+\left\|u_{n}\right\|\right) I^{\prime}\left(u_{n}\right) \longrightarrow 0$, as $\longrightarrow \infty,\left\{u_{n}\right\}$ is bounded in $X_{0}$, we have 


$$
\begin{gathered}
\left|\left\langle I^{\prime}\left(u_{n}\right), u_{n}-u\right\rangle\right| \leq \mid\left\langle I^{\prime}\left(u_{n}, u_{n}\right\rangle|+|\left\langle I^{\prime}\left(u_{n}, u\right\rangle\right|\right. \\
\quad \leq\left\|I^{\prime}\left(u_{n}\right)\right\|_{X_{0}}\left\|u_{n}\right\|_{X_{0}}+\left\|I^{\prime}\left(u_{n}\right)\right\|_{X_{0}}\|u\|_{X_{0}},
\end{gathered}
$$

therefore, we obtain

$$
\lim _{n \longrightarrow \infty}\left\langle I^{\prime}\left(u_{n}\right), u_{n}-u\right\rangle=0
$$

or

$$
\begin{aligned}
& \left\langle I^{\prime}\left(u_{n}\right), u_{n}-u\right\rangle \\
& =\left(a-b \int_{\Omega \times \Omega} \frac{\left|u_{n}(x)-u_{n}(y)\right|^{p(x, y)}}{p(x, y)|x-y|^{N+s p(x, y)}} d x d y\right) \\
& \quad \times \int_{\Omega \times \Omega} \frac{\left|u_{n}(x)-u_{n}(y)\right|^{p(x, y)-2}\left(u_{n}(x)-u_{n}(y)-u(x)+u(y)\right)}{|x-y|^{N+s p(x, y)}} d x d y \\
& \quad-\lambda \int_{\Omega}\left|u_{n}\right|^{q(x)-2} u_{n}\left(u_{n}-u\right) d x-\int_{\Omega} f\left(x, u_{n}\right)\left(u_{n}-u\right) d x,
\end{aligned}
$$

and we deduce from Lemma 13 that

$$
\begin{aligned}
& \left(a-b \int_{\Omega \times \Omega} \frac{\left|u_{n}(x)-u_{n}(y)\right|^{p(x, y)}}{p(x, y)|x-y|^{N+s p(x, y)}} d x d y\right) \\
& \quad \times \int_{\Omega \times \Omega} \frac{\left|u_{n}(x)-u_{n}(y)\right|^{p(x, y)-2}\left(u_{n}(x)-u_{n}(y)-u(x)+u(y)\right)}{|x-y|^{N+s p(x, y)}} d x d y \longrightarrow 0 .
\end{aligned}
$$

Since $\left\{u_{n}\right\}$ is bounded in $X_{0}$, passing to a subsequence, we may assume

$$
\int_{\Omega \times \Omega} \frac{\left|u_{n}(x)-u_{n}(y)\right|^{p(x, y)}}{p(x, y)|x-y|^{N+s p(x, y)}} d x d y \longrightarrow t_{0} \geq 0 \text { as } n \longrightarrow \infty .
$$

(i) If $t_{0}=0$, then $u_{n}$ strongly converges to $u=0$ in $X_{0}$

(ii) If $t_{0}>0, t_{0} \neq a / b$, then

$$
a-b \int_{\Omega \times \Omega} \frac{\left|u_{n}(x)-u_{n}(y)\right|^{p(x, y)}}{p(x, y)|x-y|^{N+s p(x, y)}} d x d y \longrightarrow 0
$$

is not true. Because $\left\{u_{n}\right\}$ is bounded in $X_{0}$, so,

$$
\left\{a-b \int_{\Omega \times \Omega} \frac{\left|u_{n}(x)-u_{n}(y)\right|^{p(x, y)}}{p(x, y)|x-y|^{N+s p(x, y)}} d x d y\right\}
$$

is bounded. (iii) If $t_{0}>0, \mathrm{t}_{0}=a / b$, then $a-b \int_{\Omega \times \Omega}\left(\left|u_{n}(x)-u_{n}(y)\right|^{p(x, y)}\right.$ $\left.|p(x, y)| x-\left.y\right|^{N+s p(x, y)}\right) d x d y \longrightarrow 0$. We define a function by

$$
\psi(u)=\lambda \int_{\Omega} \frac{1}{q(x)}|u|^{q(x)} d x+\int_{\Omega} F(x, u) d x, \text { for all } u \in X_{0},
$$

and then

$$
\left\langle\psi^{\prime}(u), \varphi\right\rangle=\lambda \int_{\Omega}|u|^{q(x)-2} u \varphi d x+\int_{\Omega} f(x, u) \varphi d x \text {, for all } u, \varphi \in X_{0},
$$

and it follows that

$$
\begin{aligned}
\left\langle\psi^{\prime}\left(u_{n}\right)-\psi^{\prime}(u), \varphi\right\rangle= & \lambda \int_{\Omega}\left(\left|u_{n}\right|^{q(x)-2} u_{n}-|u|^{q(x)-2} u\right) \varphi d x \\
& +\int_{\Omega}\left(f\left(x, u_{n}\right)-f(x, u)\right) \varphi d x .
\end{aligned}
$$

Since $u_{n} \longrightarrow u$ in $L^{q(x)}(\Omega)$, which implies that

$$
\left|u_{n}\right|^{q(x)-2} u_{n} \longrightarrow|u|^{q(x)-2} u \text { in } L^{q(x) / q(x)-1}(\Omega),
$$

according to the Hölder's inequality, we get

$$
\begin{aligned}
& \left|\int_{\Omega}\left(\left|u_{n}\right|^{q(x)-2} u_{n}-|u|^{q(x)-2} u\right) \varphi d x\right| \\
& \quad \leq\left.\int_{\Omega}|| u_{n}\right|^{q(x)-2} u_{n}-|u|^{q(x)-2} u|| \varphi \mid d x \\
& \quad \leq\left.|| u_{n}\right|^{q(x)-2} u_{n}-\left.|u|^{q(x)-2} u\right|_{q(x) / q(x)-1}|\varphi|_{q(x)} \longrightarrow 0 .
\end{aligned}
$$

Hence, we obtain

$$
\int_{\Omega}\left(\left|u_{n}\right|^{q(x)-2} u_{n}-|u|^{q(x)-2} u\right) \varphi d x=0 .
$$

With a slight modification of the above proof, we can also prove the following conclusion; so, we have omitted the details. By Hölder's inequality, (38) and (42), we deduce that

$$
\lim _{n \longrightarrow \infty} \int_{\Omega}\left|f\left(x, u_{n}\right)-f(x, u) \| \varphi\right| d x=0 .
$$

Combining with (65) and (66), we get

$$
\left\|\psi^{\prime}\left(u_{n}\right)-\psi^{\prime}(u)\right\|_{X_{0}^{*}} \longrightarrow 0 \text { and } \psi^{\prime}\left(u_{n}\right) \longrightarrow \psi^{\prime}(u) .
$$


While

$$
\begin{aligned}
\left\langle I^{\prime}\left(u_{n}\right), \varphi\right\rangle= & \left(a-b \int_{\Omega \times \Omega} \frac{\left|u_{n}(x)-u_{n}(y)\right|^{p(x, y)}}{p(x, y)|x-y|^{N+s p(x, y)}} d x d y\right) \\
& \times \int_{\Omega \times \Omega} \frac{\left|u_{n}(x)-u_{n}(y)\right|^{p(x, y)-2}\left(u_{n}(x)-u_{n}(y)\right)(\varphi(x)-\varphi(y))}{|x-y|^{N+s p(x, y)}} d x d y \\
& -\left\langle\psi^{\prime}\left(u_{n}\right), \varphi\right\rangle,
\end{aligned}
$$

since $\left\langle I^{\prime}\left(u_{n}\right), \varphi\right\rangle \longrightarrow 0$, and $a-b \int_{\Omega \times \Omega}\left(\left|u_{n}(x)-u_{n}(y)\right|^{p(x, y)} /\right.$ $\left.p(x, y)|x-y|^{N+s p(x, y)}\right) d x d y \longrightarrow 0$, it follows that $\psi^{\prime}\left(u_{n}\right) \longrightarrow$ 0 as $n \longrightarrow \infty$, i.e., by virtue of (61), we obtain

$$
-\lambda|u|^{q(x)-2} u-f(x, u)=0 \text {, a.e. } x \in \Omega
$$

by the fundamental lemma of the variational method (see [43]). It follows that $u=0$. So,

$$
\begin{aligned}
\psi\left(u_{n}\right)= & \lambda \int_{\Omega} \frac{1}{q(x)}\left|u_{n}\right|^{q(x)} d x+\int_{\Omega} F\left(x, u_{n}\right) d x \rightarrow \lambda \int_{\Omega} \frac{1}{q(x)}|u|^{q(x)} d x \\
& +\int_{\Omega} F(x, u) d x=0 .
\end{aligned}
$$

Hence, for $t_{0}=a / b$, we have

$$
\begin{aligned}
I\left(u_{n}\right)= & a \int_{\Omega \times \Omega} \frac{\left|u_{n}(x)-u_{n}(y)\right|^{p(x, y)}}{p(x, y)|x-y|^{N+s p(x, y)}} d x d y \\
& -\frac{b}{2}\left(\int_{\Omega \times \Omega} \frac{\left|u_{n}(x)-u_{n}(y)\right|^{p(x, y)}}{p(x, y)|x-y|^{N+s p(x, y)}} d x d y\right)^{2} \\
& -\lambda \int_{\Omega} \frac{1}{q(x)}\left|u_{n}\right|^{q(x)} d x-\int_{\Omega} F\left(x, u_{n}\right) d x \longrightarrow \frac{a^{2}}{2 b} .
\end{aligned}
$$

This is a contradiction with $I\left(u_{n}\right) \longrightarrow c<a^{2} / 2 b$; then, $a$ $-b \int_{\Omega \times \Omega}\left(\left|u_{n}(x)-u_{n}(y)\right|^{p(x, y)} / p(x, y)|x-y|^{N+s p(x, y)}\right) d x d y$

$\longrightarrow 0$ is not true and similarly to (ii), and we obtain

$$
\left\{a-b \int_{\Omega \times \Omega} \frac{\left|u_{n}(x)-u_{n}(y)\right|^{p(x, y)}}{p(x, y)|x-y|^{N+s p(x, y)}} d x d y\right\}
$$

that is bounded. So, it can be concluded from the above discussion (ii) and (iii) that

$$
\int_{\Omega \times \Omega} \frac{\left|u_{n}(x)-u_{n}(y)\right|^{p(x, y)-2}\left(u_{n}(x)-u_{n}(y)-u(x)+u(y)\right)}{|x-y|^{N+s p(x, y)}} d x d y \longrightarrow 0 .
$$

Thus, invoking the $S_{+}$condition (see (2) of Lemma 7), we deduce that the $\left\{u_{n}\right\}$ converges to $u$ in $X_{0}$.

Therefore, the energy functional $I$ satisfies the $(\mathrm{Ce})_{c}$ condition.
Lemma 15. Let the function $f$ satisfy $(f 1),(f 3)$, and (f4), and then the energy functional I satisfies the $(\mathrm{Ce})_{c}^{*}$ condition, where precisely $c<a^{2} / 2 b$.

Proof. Consider a sequence $\left\{u_{n_{j}}^{-}\right\}_{n_{j}} \subset X_{0}$ such that $u_{n_{j}}^{-} \in A_{n_{j}}$, $I\left(u_{n_{j}}^{-}\right) \longrightarrow c,\left.I^{\prime}\left(u_{n_{j}}^{-}\right)\right|_{A_{n_{j}}} ^{\prime} \longrightarrow 0$, in $X_{0}^{*}$, as $n_{j} \longrightarrow \infty$. By the same method used in the proof of Lemma 14, we can prove that $\left\{u_{n_{j}}^{-}\right\}_{n_{j}}$ has a strongly convergent subsequence in $X_{0}$. The details are omitted.

Remark 16. Since the $(\mathrm{Ce})_{c}$ and $(\mathrm{Ce})_{c}^{*}$ conditions hold for the energy $c<a^{2} / 2 b$, we discuss the negative energy solutions for the problem $\left(P_{\lambda}\right)$.

\section{Proof of Theorem 2}

We firstly prove two lemmas.

Lemma 17. Let assumptions (f1), $(f 2)$, and (f3) hold, and then there are constants $\rho, \lambda^{*}$ and $\alpha>0$ such that $I(u) \geq \alpha$, where $\lambda \in\left(0, \lambda^{*}\right)$ and $\|u\|_{X_{0}}=\rho$.

Proof. For any $\varepsilon \in(0,1)$ and $\lambda>0$, by (f1) and (f2), there exists $C_{\varepsilon}>0$ such that

$$
|F(x, u)| \leq \frac{\varepsilon}{p^{+}}|u|^{p+}+\frac{C_{\varepsilon}}{r(x)}|u|^{r(x)} .
$$

Let $u \in X_{0}$ be such that $\|u\|=\rho \in(0,1)$ and combining with (74), we deduce that

$$
\begin{aligned}
I(u)= & \int_{\Omega \times \Omega} \frac{|u(x)-u(y)|^{p(x, y)}}{p(x, y)|x-y|^{N+s p(x, y)}} d x d y \\
& -\frac{b}{2}\left(\int_{\Omega \times \Omega} \frac{|u(x)-u(y)|^{p(x, y)}}{p(x, y)|x-y|^{N+s p(x, y)}} d x d y\right)^{2} \\
& -\lambda \int_{\Omega} \frac{1}{q(x)}|u|^{q(x)} d x-\int_{\Omega} F(x, u) d x \\
\geq & \int_{\Omega \times \Omega} \frac{|u(x)-u(y)|^{p(x, y)}}{p(x, y)|x-y|^{N+s p(x, y)}} d x d y \\
& -\frac{b}{2}\left(\int_{\Omega \times \Omega} \frac{|u(x)-u(y)|^{p(x, y)}}{p(x, y)|x-y|^{N+s p(x, y)}} d x d y\right)^{2} \\
& -\lambda \int_{\Omega} \frac{1}{q(x)}|u|^{q(x)} d x-\int_{\Omega} \frac{\varepsilon}{p^{+}}|u|^{p^{+}} d x \\
& -\int_{\Omega} \frac{C_{\varepsilon}}{r(x)}|u|^{r(x)} d x \geq \frac{a}{p^{+}} \rho_{p(x . y)}(u)-\frac{b}{2 p^{-2}} \rho_{p(x . y)}^{2}(u) \\
& -\frac{\lambda}{q^{-}} \rho_{q(x)}(u)-\frac{\varepsilon}{p^{+}} \rho_{p^{+}}(u)-\frac{C_{\varepsilon}}{r^{-}} \rho_{r(x)}(u) .
\end{aligned}
$$

By Theorem 6, Lemma 5, and Lemma 8, there exist positive constants $C_{q^{-}}, C_{p}, C_{r}$ such that 


$$
|u|_{q(x)}^{q^{-}} \leq C_{q^{-}}\|u\|_{X_{0}}^{q^{-}},|u|_{p^{+}}^{p^{+}} \leq C_{p}\|u\|_{X_{0}}^{p^{+}},|u|_{r(x)}^{r^{-}} \leq C_{r}\|u\|_{X_{0}}^{r^{-}} .
$$

Hence, we obtain

$$
\begin{aligned}
I(u) \geq & \frac{a}{p^{+}}\|u\|_{X_{0}}^{p^{+}}-\frac{b}{2 p^{-2}}\|u\|_{X_{0}}^{2 p^{-}}-\frac{\lambda C_{q^{-}}}{q^{-}}\|u\|_{X_{0}}^{q^{-}}-\frac{\varepsilon C_{p}}{p^{+}}\|u\|_{X_{0}}^{p^{+}} \\
& -\frac{C_{\varepsilon} C_{r}}{r^{-}}\|u\|_{X_{0}}^{r^{-}}=\|u\|_{X_{0}}^{p^{+}}\left(\frac{a}{p^{+}}-\frac{b}{2 p^{-2}}\|u\|_{X_{0}}^{2 p^{-}-p^{+}}\right. \\
& \left.-\frac{\lambda C_{q^{-}}}{q^{-}}\|u\|_{X_{0}}^{q^{-}-p^{+}}-\frac{\varepsilon C_{p}}{p^{+}}-\frac{C_{\varepsilon} C_{r}}{r^{-}}\|u\|_{X_{0}}^{r^{-}-p^{+}}\right) .
\end{aligned}
$$

Choose $\varepsilon=a / 2 C_{p}>0$ and take

$$
I(t)=\frac{a}{2 p^{+}}-\frac{b}{2 p^{-2}} t^{2 p^{-}-p^{+}}-\frac{C_{\varepsilon} C_{r}}{r^{-}} t^{r^{-}-p^{+}}, \text {for all } t \geq 0 .
$$

Note that $p^{+}<2 p^{-}<r^{-}$(see (11)), and we can conclude that there exists a constant $\rho>0$ such that

$$
I(\rho)=\max _{t \geq 0} I(t)>0 .
$$

Therefore, taking $\lambda^{*}:=\left(q^{-} / 2 C_{q^{-}} \rho^{q^{-}-p^{+}}\right) I(\rho)>0$, we have

$$
I(u) \geq \frac{1}{2} \rho^{p^{+}} I(\rho)=: \alpha>0,
$$

whenever $\|u\|_{X_{0}}=\rho$ and $\lambda \in\left(0, \lambda^{*}\right)$. This completes the proof.

Lemma 18. Let assumptions (f1), (f2,) and (f3) hold, and then

$$
-\infty<\inf \left\{I(u): u \in \bar{B}_{\rho}\right\}<0
$$

where $\rho$ is given by Lemma 17 and $\bar{B}_{\rho}:=\left\{u \in X_{0}:\|u\|_{X_{0}}\right.$ $\leq \rho\}$.

Proof. By (f2) and (f3), for any $M_{1}>0$, there exists $C_{M_{1}}>0$ such that

$$
F(x, u) \geq M_{1}|u|^{2 p^{+}}-C_{M_{1}}|u|^{p^{+}}, \text {for all }(\mathrm{x}, \mathrm{u}) \in \Omega \times \mathbb{R} .
$$

Let $\phi \in X_{0}, \phi>0$ and $t>0$, combining with (82), we have

$$
\begin{aligned}
I(t \phi)= & a \int_{\Omega \times \Omega} \frac{|t \phi(x)-t \phi(y)|^{p(x, y)}}{p(x, y)|x-y|^{N+s p(x, y)}} d x d y \\
& -\frac{b}{2}\left(\int_{\Omega \times \Omega} \frac{|t \phi(x)-t \phi(y)|^{p(x, y)}}{p(x, y)|x-y|^{N+s p(x, y)}} d x d y\right)^{2} \\
& -\lambda \int_{\Omega} \frac{1}{q(x)}|t \phi|^{q(x)} d x-\int_{\Omega} F(x, t \phi) d x \\
\leq & a \int_{\Omega \times \Omega} \frac{|t \phi(x)-t \phi(y)|^{p(x, y)}}{p(x, y)|x-y|^{N+s p(x, y)}} d x d y \\
& -\frac{b}{2}\left(\int_{\Omega \times \Omega} \frac{|t \phi(x)-t \phi(y)|^{p(x, y)}}{p(x, y)|x-y|^{N+s p(x, y)}} d x d y\right)^{2} \\
& -\lambda \int_{\Omega} \frac{1}{q(x)}|t \phi|^{q(x)} d x-\int_{\Omega} M_{1}|t \phi|^{2 p^{+}} d x \\
& +\int_{\Omega} C_{M_{1}}|t \phi|^{p^{-}} d x \leq \frac{a t^{p^{-}}}{p^{-}} \int_{\Omega \times \Omega} \frac{|\phi(x)-\phi(y)|^{p(x, y)}}{|x-y|^{N+s p(x, y)}} d x d y \\
& -\frac{b t^{2 p^{+}}}{2 p^{+2}}\left(\int_{\Omega \times \Omega} \frac{|\phi(x)-\phi(y)|^{p(x, y)}}{|x-y|^{N+s p(x, y)}} d x d y\right)^{2} \\
& -\frac{\lambda t^{q^{+}}}{q^{+}} \int_{\Omega}^{|\phi|^{q(x)} d x-M_{1} t^{2 p^{+}}} \int_{\Omega}|\phi|^{2 p^{+}} d x+C_{M_{1}} t^{p^{+}} \int_{\Omega}|\phi|^{p^{+}} d x .
\end{aligned}
$$

Since $q^{+}<p^{-}<p^{+}$(see (11)), for $t>0$, it is small enough. Hence, we obtain that

$$
-\infty<\inf \left\{I(u): u \in \bar{B}_{\rho}\right\}<0,
$$

where $\rho$ is given by Lemma 17 and $\bar{B}_{\rho}:=\left\{u \in X_{0}:\|u\|_{X_{0}} \leq \rho\right\}$

Now, we give the proof of Theorem 2 .

Proof. By Lemma 17 and Lemma 18, we know that

$$
-\infty<c=\inf _{\bar{B}_{\rho}} I(u)<0<\alpha<\inf _{\partial B_{\rho}} I(u)
$$

whenever $\lambda \in\left(0, \lambda^{*}\right)$. Set

$$
\frac{1}{n} \in\left(0, \inf _{\partial B_{\rho}} I(u)-\inf _{\bar{B}_{\rho}} I(u)\right), n \in \mathbb{N} .
$$

So, by Theorem 9 (Ekeland's variational principle), there exists $u_{n} \in \bar{B}_{\rho}$ such that

$$
c \leq I\left(u_{n}\right) \leq c+\frac{1}{n},
$$

$$
I\left(u_{n}\right) \leq I(u)+\frac{1}{n}\left\|u_{n}-u\right\|_{X_{0}},
$$


for all $u \in \bar{B}_{\rho}, u_{n} \neq u$. In that way, by (86)-(88), we have

$$
I\left(u_{n}\right) \leq c+\frac{1}{n} \leq \inf _{u \in \bar{B}_{\rho}} I(u)+\frac{1}{n}<\inf _{u \in \partial B_{\rho}} I(u),
$$

so that $u_{n} \in B_{\rho}$.

Define the functional $M_{n}: \bar{B}_{\rho} \longrightarrow \mathbb{R}$ by

$$
M_{n}(u)=I(u)+\frac{1}{n}\left\|u_{n}-u\right\|_{X_{0}}, \text { for all } u \in \bar{B}_{\rho}
$$

Like that, (88) implies that $M_{n}\left(u_{n}\right)<M_{n}(u)$ for all $u \in \bar{B}_{\rho}$, with $u_{n} \neq u$, and thus $u_{n}$ is a strict local minimum of $M_{n}$. Furthermore, for all $v \in B_{1}$, where $B_{1}=\left\{v \in X_{0}:\|v\|_{X_{0}}=1\right\}$, taking $t>0$ small enough, we have

$$
\frac{M_{n}\left(u_{n}+t v\right)-M_{n}\left(u_{n}\right)}{t} \geq 0
$$

which implies that

$$
\frac{I\left(u_{n}+t v\right)-I\left(u_{n}\right)}{t}+\frac{1}{n}\|v\|_{X_{0}} \geq 0
$$

Thus,

$$
\left\langle I^{\prime}\left(u_{n}\right), v\right\rangle+\frac{1}{n}\|v\|_{X_{0}} \geq 0 \text { for all } v \in B_{1} .
$$

In the above inequality, replacing $v$ with $-v$, we have

$$
-\left\langle I^{\prime}\left(u_{n}\right), v\right\rangle+\frac{1}{n}\|v\|_{X_{0}} \geq 0 \text { for all } v \in B_{1}
$$

Hence,

$$
\left\|I^{\prime}\left(u_{n}\right)\right\|_{X_{0}^{*}} \leq \frac{1}{n}
$$

Passing to the limit in (87) and (95), we conclude that there is a sequence $\left\{u_{n}\right\} \subset \bar{B}_{\rho}$ such that

$$
I\left(u_{n}\right) \longrightarrow c<0 \text { and }\left\|I^{\prime}\left(u_{n}\right)\right\|_{X_{0}^{*}} \longrightarrow 0, \text { asn } \longrightarrow \infty .
$$

To sum up, by Lemma 14, there exists a strongly convergent sequence $\left\{u_{n}\right\}$, still denoted by $\left\{u_{n}\right\}$, such that $u_{n} \longrightarrow$ $u_{0}$ in $X_{0}$. Consequently, $u_{0}$ is a solution of $\left(P_{\lambda}\right)$, with $I\left(u_{0}\right)$ $<0$. The proof of Theorem 2 is completed.

\section{Proof of Theorem 3}

In order to prove Theorem 3, we need the following result.
Lemma 19 (see [44], Lemma 18). Assume that $\zeta(x) \in C_{+}(\bar{\Omega}$ ),$\zeta(x)<p^{*}(x)$, for any $x \in \bar{\Omega}$ and denote

$$
\beta_{k}=\sup _{u \in B_{k},\|u\|_{X_{0}}=1}|u|_{\zeta(x)},
$$

then $\lim _{k \rightarrow \infty} \beta_{k}=0$.

We give the proof of Theorem 3 .

Proof. The function $f$ satisfies hypotheses (f1)-(f5), and it is obvious that $I(-u)=I(u)$ for all $u \in X_{0}$. Together with Lemma 15 , we only need to verify that the energy functional $I: X_{0} \longrightarrow \mathbb{R}$ satisfies all conditions (B1)-(B3) of Theorem 10 .

Step 1. Verification of condition (B1). According to (f1) and (f2), there exist $C_{2}>0, C_{3}>0$, such that

$$
|F(x, u)| \leq \frac{C_{2}}{p^{+}}|u|^{p^{+}}+\frac{C_{3}}{r(x)}|u|^{r(x)} .
$$

For any $u \in B_{k}$ with $\|u\| \leq \min \left(1,\left(1 / C_{0}\right)\right)$, combining with (98) Theorem 6 and Lemma 8, there exist positive constants $C_{q^{-}}, C_{p}, C_{r}$ such that we obtain

$$
\begin{aligned}
I(u)= & \int_{\Omega \times \Omega} \frac{|u(x)-u(y)|^{p(x, y)}}{p(x, y)|x-y|^{N+s p(x, y)}} d x d y \\
& -\frac{b}{2}\left(\int_{\Omega \times \Omega} \frac{|u(x)-u(y)|^{p(x, y)}}{p(x, y)|x-y|^{N+s p(x, y)}} d x d y\right)^{2} \\
& -\lambda \int_{\Omega} \frac{1}{q(x)}|u|^{q(x)} d x-\int_{\Omega} F(x, u) d x \\
\geq & \int_{\Omega \times \Omega} \frac{|u(x)-u(y)|^{p(x, y)}}{p(x, y)|x-y|^{N+s p(x, y)}} d x d y \\
& -\frac{b}{2}\left(\int_{\Omega \times \Omega} \frac{|u(x)-u(y)|^{p(x, y)}}{p(x, y)|x-y|^{N+s p(x, y)}} d x d y\right)^{2} \\
& -\lambda \int_{\Omega} \frac{1}{q(x)}|u|^{q(x)} d x-\int_{\Omega} \frac{C_{2}}{p^{+}}|u|^{p^{+}} d x \\
& -\int_{\Omega} \frac{C_{3}}{r(x)}|u|^{r(x)} d x \geq \frac{a}{p^{+}} \rho_{p(x . y)}(u)-\frac{b}{2 p^{-2}} \rho_{p(x . y)}^{2}(u) \\
& -\frac{\lambda}{q^{-}} \rho_{q(x)}(u)-\frac{C_{2}}{p^{+}} \rho_{p^{+}}(u)-\frac{C_{3}}{r^{-}} \rho_{r(x)}(u) \geq \frac{a}{p^{+}}\|u\|_{X_{0}}^{p^{+}} \\
& -\frac{b}{2 p^{-2}}\|u\|_{X_{0}}^{2 p^{-}}-\frac{\lambda C_{q^{-}}}{q^{-}}\|u\|_{X_{0}}^{q^{-}}-\frac{C_{2} C_{p}}{p^{+}}\|u\|_{X_{0}}^{p^{+}} \\
& -\frac{C_{3} C_{r}}{r^{-}}\|u\|_{X_{0}}^{r^{-}} \cdot
\end{aligned}
$$

According to Lemma 19 and the conditions $q^{-}<p^{+}<$ $\sqrt{2} p^{-}$(see (12)), it follows that 


$$
\begin{aligned}
I(u) \geq & \frac{a}{p^{+}}\|u\|_{X_{0}}^{p^{+}}-\frac{b}{2 p^{-2}}\|u\|_{X_{0}}^{2 p^{-}}-\frac{\lambda C_{q^{-}} \beta_{k}^{q^{-}}}{q^{-}}\|u\|_{X_{0}}^{q^{-}} \\
& -\frac{C_{2} C_{p} \beta_{k}{ }^{p^{+}}}{p^{+}}\|u\|_{X_{0}}^{p^{+}}-\frac{C_{3} C_{r} \beta_{k}^{r^{-}}}{r^{-}}\|u\|_{X_{0}}^{r^{-}} \\
\geq & \left(\frac{a}{p^{+}}-\frac{b}{2 p^{-2}}\right)\|u\|_{X_{0}}^{2 p^{-}}-\frac{\left(\lambda C_{q^{-}}+C_{2} C_{p}\right) \beta_{k}{ }^{q^{-}}}{q^{-}}\|u\|_{X_{0}}^{q^{-}} \\
& -\frac{C_{3} C_{r} \beta_{k}^{r^{-}}}{r^{-}}\|u\|_{X_{0}}^{r^{-}},
\end{aligned}
$$

since $a \geq b$ and $p^{+}<\sqrt{2} p^{-}<2 p^{-}<r^{-}$(see (12)) imply that $a / p^{+}-b / 2 p^{-2}=2 p^{-2} a-b p^{+} / 2 p^{-2} p^{+}>0$.

Hence, we deduce

$$
\begin{aligned}
I(u) \geq & \left(\frac{2 p^{-2} a-b p^{+}}{2 p^{-2} p^{+}}-\frac{C_{3} C_{r} \beta_{k}^{r^{-}}}{r^{-}}\right)\|u\|_{X_{0}}^{r^{-}} \\
& -\frac{\left(\lambda C_{q^{-}}+C_{2} C_{p}\right) \beta_{k}^{q^{-}}}{q^{-}}\|u\|_{X_{0}}^{q^{-}} .
\end{aligned}
$$

Choosing $2 p^{-2} a-b p^{+} / 4 p^{-2} p^{+}>C_{3} C_{r} \beta_{k}{ }^{r^{-}} / r^{-}$, we have

$$
I(u) \geq \frac{2 p^{-2} a-b p^{+}}{4 p^{-2} p^{+}}\|u\|_{X_{0}}^{r^{-}}-\frac{\left(\lambda C_{q^{-}}+C_{2} C_{p}\right) \beta_{k}^{q^{-}}}{q^{-}}\|u\|_{X_{0}}^{q^{-}} .
$$

Obviously, there exists a large enough $k$ such that

$$
I(u) \geq\|u\|_{X_{0}}^{q^{-}}\left(\frac{2 p^{-2} a-b p^{+}}{4 p^{-2} p^{+}}\|u\|_{X_{0}}^{r^{-}-q^{-}}-\frac{\left(\lambda C_{q^{-}}+C_{2} C_{p}\right) \beta_{k}^{q^{-}}}{q^{-}}\right) .
$$

We choose

$$
\rho_{k}:=\left(\frac{4\left(\lambda C_{q^{-}}+C_{2} C_{p}\right) p^{+} p^{-2}}{q^{-} 2 a p^{-2}-b q^{-} p^{+}} \beta_{k}^{q^{-}}\right)^{1 / r^{-}-q^{-}}
$$

by Lemma 19, and we know that $\rho_{k} \longrightarrow 0$ as $k \longrightarrow \infty$. So, there exists $k_{0}>0$, such that $k>k_{0}$ and $u \in B_{k}$ with $\|u\|_{X_{0}}=$ $\rho_{k}$, and we have $I(u) \geq 0$. Therefore, the condition (B1) of Theorem 10 holds.

Step 2. Verification of condition (B2). By (f2) and (f3), there exist $C_{4}, C_{5}>0$ such that

$$
F(x, u) \geq C_{4}|u|^{2 p^{+}}-C_{5}|u|^{p^{+}}, \text {for all }(\mathrm{x}, \mathrm{u}) \in \Omega \times \mathbb{R} .
$$

For $u \in A_{k}$, when $\|u\|=\gamma_{k}$ with $\rho_{k}>\gamma_{k}>0$, we have

$$
\begin{aligned}
I(u)= & a \int_{\Omega \times \Omega} \frac{|u(x)-u(y)|^{p(x, y)}}{p(x, y)|x-y|^{\mathrm{N}+s p(x, y)}} d x d y \\
& -\frac{b}{2}\left(\int_{\Omega \times \Omega} \frac{|u(x)-u(y)|^{p(x, y)}}{p(x, y)|x-y|^{N+s p(x, y)}} d x d y\right)^{2} \\
& -\lambda \int_{\Omega} \frac{1}{q(x)}|u|^{q(x)} d x-\int_{\Omega} F(x, u) d x \\
\leq & a \int_{\Omega \times \Omega} \frac{|u(x)-u(y)|^{p(x, y)}}{p(x, y)|x-y|^{N+s p(x, y)}} d x d y \\
& -\frac{b}{2}\left(\int_{\Omega \times \Omega} \frac{|u(x)-u(y)|^{p(x, y)}}{p(x, y)|x-y|^{N+s p(x, y)}} d x d y\right)^{2} \\
& -\lambda \int_{\Omega} \frac{1}{q(x)}|u|^{q(x)} d x-\int_{\Omega} C_{4}|u|^{2 p^{+}} d x+\int_{\Omega} C_{5}|u|^{p^{+}} d x \\
\leq & \frac{a}{p^{-}} \rho_{p(x, y)}(u)-\frac{b}{2 p^{+2}} \rho_{p(x, y)}^{2}(u)-\frac{\lambda}{q^{+}} \rho_{q(x)}(u) \\
& -C_{4} \rho_{2 p^{+}}(u)+C_{5} \rho_{p^{+}}(u) .
\end{aligned}
$$

Since all norms on the finite-dimensional space $A_{k}$ are equivalent, we obtain

$$
\begin{aligned}
I(u) \leq & \frac{a}{p^{-}}\|u\|_{X_{0}}^{p^{-}}-\left(\frac{b}{2 p^{+2}}+C_{4} C_{2 p^{+}}\right)\|u\|_{X_{0}}^{2 p^{+}}-\frac{\lambda C_{q^{+}}}{q^{+}}\|u\|_{X_{0}}^{q^{+}} \\
& +C_{5} C_{p^{+}}\|u\|_{X_{0}}^{p^{+}} .
\end{aligned}
$$

According to the condition $q^{+}<p^{-}<p^{+}$(see (12)), for some $\gamma_{k}=\|u\|_{X_{0}}$ that is small enough, we deduce that

$$
b_{k}=\max _{u \in A_{k},\|u\|_{X_{0}}=\gamma_{k}} I(u) \leq 0 .
$$

Therefore, the condition (B2) of Theorem 10 holds.

Step 3. Verification of condition (B3). By verification of condition (B1), one has that for $k \geq k_{0}$ and $u \in B_{k}$ with $\|u(x)\|_{X_{0}} \leq \gamma_{k}$,

$$
I(u) \geq-\frac{\left(\lambda+C_{2} C_{p}\right) \beta_{k}{ }^{q^{-}}}{q^{-}}\|u\|_{X_{0}}^{q^{-}} \geq-\frac{\left(\lambda+C_{2} C_{p}\right) \beta_{k}{ }^{q^{-}}}{q^{-}} \gamma_{k}^{q^{-}},
$$

since $\gamma_{k} \longrightarrow 0$ as $k \longrightarrow \infty$. Therefore, the condition (B3) of Theorem 10 also holds.

By above three steps and Lemma 15, I satisfies all conditions of Theorem 10. The proof of Theorem 3 is completed.

\section{Data Availability}

The data used to support the findings of this study are available from the corresponding author upon request. 


\section{Conflicts of Interest}

The authors declare that they have no competing interests.

\section{Authors' Contributions}

Each of the authors contributed to each part of this study equally, and all authors read and approved the final manuscript.

\section{Acknowledgments}

This work is supported by the Fundamental Research Funds for the Central Universities (2019B44914), Natural Science Foundation of Jiangsu Province (BK20180500), the National Key Research and Development Program of China (2018YFC1508100), and Natural Science Foundation of China (11701595).

\section{References}

[1] F. Abdolrazaghi and A. Razani, "On the weak solutions of an overdetermined system of nonlinear fractional partial integro-differential equations," Miskolc Mathematical Notes, vol. 20, no. 1, pp. 3-16, 2019.

[2] F. Abdolrazaghi and A. Razani, "A unique weak solution for a kind of coupled system of fractional Schrödinger equations," Opuscula Mathematica, vol. 40, no. 3, pp. 313-322, 2020.

[3] G. M. Bisci, V. D. Rădulescu, and R. Servadei, Variational methods for nonlocal fractional problems, vol. 162, Cambridge University Press, 2016.

[4] M. Makvand Chaharlang and A. Razani, "A fourth order singular elliptic problem involving \$p\$-biharmonic operator," Taiwanese Journal of Mathematics, vol. 23, no. 3, pp. 589599, 2018.

[5] L. Caffarelli and L. Silvestre, "An extension problem related to the fractional Laplacian," Communications in Partial Differential Equations, vol. 32, no. 8, pp. 1245-1260, 2007.

[6] A. Iannizzotto, S. Liu, K. Perera, and M. Squassina, "Existence results for fractional p-Laplacian problems via Morse theory," Advances in Calculus of Variations, vol. 9, no. 2, pp. 101-125, 2014.

[7] E. Lindgren and P. Lindqvist, "Fractional eigenvalues," Calculus of Variations and Partial Differential Equations, vol. 49, no. 1-2, pp. 795-826, 2014.

[8] S. Mosconi, K. Perera, M. Squassina, and Y. Yang, "The BrezisNirenberg problem for the fractional p-Laplacian," Calculus of Variations and Partial Differential Equations, vol. 55, no. 4, article 1035, pp. 1-25, 2016.

[9] J. Zuo, T. An, L. Yang, and X. Ren, “The Nehari manifold for a fractional p-Kirchhoff system involving sign-changing weight function and concave-convex nonlinearities," Journal of Function Spaces, vol. 2019, no. 1, Article ID 7624373, pp. 1-9, 2019.

[10] F. Behboudi, A. Razani, and M. Oveisiha, "Existence of a mountain pass solution for a nonlocal fractional $(p, q)$-Laplacian problem," Boundary Value Problems, vol. 2020, no. 1, 14 pages, 2020.

[11] E. di Nezza, G. Palatucci, and E. Valdinoci, "Hitchhiker's guide to the fractional Sobolev spaces," Bulletin des Sciences Mathématiques, vol. 136, no. 5, pp. 521-573, 2012.
[12] L. Del Pezzo and J. D. Rossi, "Traces for fractional Sobolev spaces with variable exponents," Advances in Operator Theory, vol. 2, no. 4, pp. 435-446, 2017.

[13] E. Guariglia, "Riemann zeta fractional derivative-functional equation and link with primes," Advances in Difference Equations, vol. 2019, no. 1, 15 pages, 2019.

[14] M. I. Abbas and M. A. Ragusa, "On the hybrid fractional differential equations with fractional proportional derivatives of a function with respect to a certain function," Symmetry, vol. 13 , no. 2 , p. $264,2021$.

[15] E. Guariglia and S. Silvestrov, "A functional equation for the Riemann zeta fractional derivative," AIP Conference Proceedings, vol. 1798, no. 1, article 020146, 2017.

[16] M. A. Ragusa, "Commutators of fractional integral operators on vanishing-Morrey spaces," Journal of Global Optimization, vol. 40, no. 1-3, pp. 361-368, 2008.

[17] C. Li, X. Dao, and P. Guo, "Fractional derivatives in complex planes," Nonlinear Analysis, vol. 71, no. 5-6, pp. 1857-1869, 2009.

[18] G. Kirchhoff, Vorlesungen über mathematische Physik, Band 1: Mechanik, B.G. Teubner, Leipzig, 1883.

[19] M. M. Chaharlang, M. A. Ragusa, and A. Razani, "A sequence of radially symmetric weak solutions for some nonlocal elliptic problem in $\mathbb{R}^{N}$," Mediterranean Journal of Mathematics, vol. 17, no. 2, pp. 1-12, 2020.

[20] C. Cowan and A. Razani, "Singular solutions of a $p$-Laplace equation involving the gradient," Journal of Differential Equations, vol. 269, no. 4, pp. 3914-3942, 2020.

[21] M. K. Hamdani, A. Harrabi, F. Mtiri, and D. D. Repovš, "Existence and multiplicity results for a new $p(x)$-Kirchhoff problem," Nonlinear Analysis, vol. 190, article 111598, 2020.

[22] M. A. Ragusa, "Elliptic boundary value problem in vanishing mean oscillation hypothesis," Commentationes Mathematicae Universitatis Carolinae, vol. 40, no. 4, pp. 651-663, 1999.

[23] A. Bahrouni, "Comparison and sub-supersolution principles for the fractional $p(x)$-Laplacian," Journal of Mathematical Analysis and Applications, vol. 458, no. 2, pp. 1363-1372, 2018.

[24] M. M. Chaharlang and A. Razani, "Two weak solutions for some Kirchhoff type problem with Neumann boundary condition," Georgian Mathematical Journal, p. 10, 2020.

[25] G. Molica Bisci and L. Vilasi, "On a fractional degenerate Kirchhoff-type problem," Communications in Contemporary Mathematics, vol. 19, no. 1, article 1550088, 2016.

[26] D. C. Liu, "On a p-Kirchhoff equation via fountain theorem and dual fountain theorem," Nonlinear Analysis: Theory, Methods \& Applications, vol. 72, no. 1, pp. 302-308, 2010.

[27] A. Fiscella and E. Valdinoci, "A critical Kirchhoff type problem involving a nonlocal operator," Nonlinear Analysis, vol. 94, pp. 156-170, 2014.

[28] Z. Liu and Z. Q. Wang, "On the Ambrosetti-Rabinowitz superlinear condition," Advanced Nonlinear Studies, vol. 4, no. 4, pp. 563-574, 2004.

[29] M. Z. Sun, "Multiple solutions of a superlinearp-Laplacian equation without AR-condition," Applicable Analysis, vol. 89, no. 3, pp. 325-336, 2010.

[30] J. B. Zuo, T. Q. An, and M. W. Li, "Superlinear Kirchhoff-type problems of the fractional $p$-Laplacian without the (AR) condition," Boundary Value Problems, vol. 2018, no. 1, Article ID 1100, 13 pages, 2018. 
[31] A. B. Zang, " $p(x)$-Laplacian equations satisfying Cerami condition," Journal of Mathematical Analysis and Applications, vol. 337, no. 1, pp. 547-555, 2008.

[32] X. Qian and W. Chao, "Existence of positive solutions for nonlocal problems with indefinite nonlinearity," Boundary Value Problems, vol. 2020, no. 1, Article ID 1343, 13 pages, 2020.

[33] G. M. Bisci, D. D. Repovš, and R. Servadei, "Nontrivial solutions of superlinear nonlocal problems," Forum Mathematicum, vol. 28, no. 6, pp. 1095-1110, 2016.

[34] D. Edmunds and J. Rákosník, "Sobolev embeddings with variable exponent," Studia Mathematica, vol. 3, no. 143, pp. 267293, 2000.

[35] X. L. Fan and D. Zhao, "On the spaces $L^{p(x)}(\Omega)$ and $W^{k, p(x)}(\Omega)$ ," Journal of Mathematical Analysis and Applications, vol. 263, no. 2, pp. 424-446, 2001.

[36] V. D. Radulescu and D. D. Repovs, Partial differential equations with variable exponents: variational methods and qualitative analysis, vol. 9, CRC Press, Boca Raton, 2015.

[37] J. Yao, "Solutions for Neumann boundary value problems involving $p(x)$-Laplace operators," Nonlinear Analysis, vol. 68, no. 5, pp. 1271-1283, 2008.

[38] L. Diening, P. Harjulehto, P. Hästö, and M. Ruzicka, "Lebesgue and Sobolev spaces with variable exponents," in Lecture Notes in Mathematics, Springer, 2011.

[39] U. Kaufmann, J. D. Rossi, and R. E. Vidal, "Fractional Sobolev spaces with variable exponents and fractional $p(x)$-Laplacians," Electronic Journal of Qualitative Theory of Differential Equations, vol. 76, no. 76, pp. 1-10, 2017.

[40] A. Bahrouni and V. T,. D. RĂdulescu, "On a new fractional Sobolev space and applications to nonlocal variational problems with variable exponent," Discrete \& Continuous Dynamical Systems - S, vol. 11, no. 3, pp. 379-389, 2018.

[41] C. Zhang and X. Zhang, "Renormalized solutions for the fractional $p(x)$-Laplacian equation with $L^{1}$ data," Nonlinear Analysis, vol. 190, article 111610, 2020.

[42] J. Mawhin and M. Willem, Critical Point Theory and Hamiltonian Systems, Springer-Verlag, New York Berlin Heidelberg, London Paris Tokyo, 1989.

[43] T. Bartsch and M. Willem, "On an elliptic equation with concave and convex nonlinearities," Proceedings of the American Mathematical Society, vol. 123, no. 11, pp. 3555-3561, 1995.

[44] X. L. Fan and Q. H. Zhang, "Existence of solutions for $p(x)$ -Laplacian Dirichlet problem," Nonlinear Analysis, vol. 52, no. 8, pp. 1843-1852, 2003. 